\title{
Heterogeneity of ERBB2 amplification in adenocarcinoma, squamous cell carcinoma and large cell undifferentiated carcinoma of the lung
}

Tobias J Grob ${ }^{1}$, Ivonne Kannengiesser ${ }^{1}$, Maria C Tsourlakis ${ }^{1}$, Djordje Atanackovic ${ }^{2}$, Alexandra M Koenig ${ }^{3}$, Yogesh K Vashist ${ }^{3}$, Hans Klose ${ }^{2}$, Andreas H Marx ${ }^{1}$, Susan Koops ${ }^{1}$, Ronald Simon ${ }^{1}$, Jakob R Izbicki ${ }^{3}$, Carsten Bokemeyer ${ }^{2}$, Guido Sauter ${ }^{1}$ and Waldemar Wilczak ${ }^{1}$

${ }^{1}$ Department of Pathology, University Medical Center Hamburg-Eppendorf, Hamburg, Germany; ${ }^{2}$ Department of Oncology/Hematology/Stem Cell Transplantation with Section Pneumology, University Cancer Center Hamburg (Hubertus-Wald Tumorzentrum), University Medical Center Hamburg-Eppendorf, Hamburg, Germany and ${ }^{3}$ Department of General, Visceral and Thoracic Surgery, University Medical Center HamburgEppendorf, Hamburg, Germany

The HER2 protein, encoded by the ERBB2 gene, is a molecular target for the treatment of breast and gastric cancer by monoclonal antibodies or tyrosine kinase inhibitors. While intratumoral heterogeneity of ERBB2 amplification is rare in breast cancer it is reported to be frequent in bladder and colorectal cancer. To address the potential heterogeneity of the HER2 status in adenocarcinomas, squamous cell carcinomas and large cell undifferentiated carcinomas of the lung, 590 tumors were analyzed for HER2 overexpression and ERBB2 amplification using FDA-approved reagents for immunohistochemistry and fluorescence in-situ hybridization (FISH). Moderate and strong immunostaining $(2+, 3+)$ was seen in $10 \%$ of the tumors. ERBB2 amplification was found in $17(3 \%)$ lung cancer patients including 10 cases $(2 \%)$ with high-level amplification forming gene clusters. ERBB2 amplification was significantly related to histologic subtype and tumor grade, resulting in $12 \%$ ERBB2 amplified tumors in the subgroup of high-grade adenocarcinomas. Heterogeneity was analyzed in all highly amplified tumors. For this purpose, all available tumor tissue blocks from these patients were evaluated. Heterogeneity of ERBB2 amplification was found in 4 of 10 tumors as assessed by FISH. These included two tumors with a mixture of low-level and high-level amplification and two tumors with non-amplified tumor areas next to regions with high-level ERBB2 amplification. High-level ERBB2 amplification occurs in a small fraction of lung cancers with a strong propensity to high-grade adenocarcinomas. Heterogeneity of amplification may limit the utility of anti-HER2 therapy in some of these tumors. Further attempts to assess the utility of HER2targeting therapy in homogeneously amplified lung cancers appear to be justified.

Modern Pathology (2012) 25, 1566-1573; doi:10.1038/modpathol.2012.125; published online 17 August 2012

Keywords: adenocarcinoma of the lung; ERBB2 gene amplification; fluorescence in-situ hybridization; HER2 expression; heterogeneity; large cell undifferentiated carcinoma of the lung; squamous cell carcinoma of the lung

The human epidermal growth factor receptor 2 gene (ERBB2, HER2/neu) is involved in the development of numerous types of human cancers and has been linked to poor prognosis in many of them. ${ }^{1-3}$ The

Correspondence: Dr TJ Grob, MD, PhD, Institute of Pathology, University Medical Center Hamburg-Eppendorf, Martinistr. 52, 20246 Hamburg, Germany.

E-mail: t.grob@uke.de

Received 17 January 2012; revised 30 May 2012; accepted 4 June 2012; published online 17 August 2012
ERBB2 gene product HER2 is the target of an antibody-based therapy (trastuzumab), which has been shown to be remarkably effective in both the metastatic and adjuvant setting for HER2-positive breast cancer $^{4-6}$ and in advanced HER2-positive gastric cancer. ${ }^{7}$ Moreover, with the tyrosine kinase inhibitor lapatinib which targets both EGFR and HER2 an alternative option for HER2-positive breast cancers has been introduced ${ }^{8}$ and is under analysis for other tumor types. 
Applying additional potent therapies would be of particular interest in tumors with a notoriously poor prognosis such as carcinomas of the lung. Preclinical cell line models suggested that ERBB2 amplification and/or overexpression of HER2 may be relevant for the development of some of these tumors and that trastuzumab has additive or synergic antitumor activity in combination with cytotoxic agents. ${ }^{9}$ HER2 overexpression in adenocarcinoma and squamous cell carcinoma of the lung as assessed by immunohistochemistry is found in $2.5-43 \%$ of cases with a clear tendency toward higher rates of positivity in adenocarcinoma than in squamous cell carcinoma. ${ }^{10-13}$ Studies assessing ERBB2 amplification found a similar variability but usually with a much lower frequency of amplified tumors. The different analytical methods such as PCR, Southern blot or fluorescence in-situ hybridization (FISH) used and the varying definitions of amplification have resulted in a wide range (1-23\%) of cases reported to be amplified. ${ }^{14-16}$ As the rate of ERBB2 amplified lung cancers is very low in studies using FDAapproved reagents and scoring criteria, it seems possible that treatment of cases with biologically less significant HER2 expression identified by immunohistochemistry, may have contributed to the poor results of HER2 targeted therapy in these tumors. ${ }^{9,17}$ Clinical trials evaluating trastuzumab therapy in lung cancer so far have included between 17 and $59 \%$ of tested patients based on immunohistochemistry. ${ }^{18,19}$

Intratumoral heterogeneity of overexpression/ amplification is another potential cause for nonresponding HER2-positive cancers. Heterogeneous ERBB2 amplification is rare in breast ${ }^{20}$ but seems to be frequent $(>50 \%)$ in bladder or colorectal cancer. ${ }^{21,22}$ Conflicting results are published on heterogeneity of ERBB2 amplification in gastric cancer. ${ }^{23-25}$ In lung cancer, heterogeneity of HER2 positivity has not been systematically analyzed. This study had the purpose to thoroughly evaluate the possible extent of HER2 heterogeneity in adenocarcinomas, squamous cell carcinomas and large cell undifferentiated carcinomas of the lung.

\section{Materials and methods}

\section{Tissues}

Two tissue microarrays were made from a total of 590 lung cancer specimens from formalin-fixed, paraffinembedded archived tissue samples of the Institute of Pathology at the University Medical Center HamburgEppendorf as described. ${ }^{26}$ Only surgical specimens of tumorectomy were used for tissue microarray construction. Tissue cores with a diameter of $0.6 \mathrm{~mm}$ were removed from the original paraffin block. All tumors were represented in duplicates on the final tissue microarrays. The usage of tissue microarrays for research purposes has been approved by the local ethics committee. The array included 219 adenocarcinomas, 250 squamous cell carcinomas and 121 undifferentiated large cell carcinomas. The median patient age at surgery was 63 years (range 36-92 years). Raw survival data were available from 290 patients. The mean follow-up period was 29 months (range 1-155 months). Survival data were obtained from the Department of General, Visceral and Thoracic Surgery, University Medical Center Hamburg-Eppendorf. All original slides of the tissues included in these tissue microarrays had been reviewed by the same pathologist determining histological type based on morphology and histological grade according to WHO $2004 .{ }^{27}$ The pathologic stage was obtained from the primary report of the Institute of Pathology. None of the arrayed tumors had been treated with anti-EGFR therapy. Consecutive, freshly cut sections of the tissue microarrays were used for FISH, immunohistochemical analysis and H\&E stained reference.

\section{ERBB2 FISH}

For proteolytic slide pretreatment, a commercial kit was utilized (paraffin pre-treatment reagent kit, Abbott Laboratories, Abbott Park, USA). For copy number detection of ERBB2, the dual color PathVysion test (Abbott Laboratories) was used according to manufacturer's instructions. A tumor was considered as high-level amplified if the ratio of average gene copy numbers and centromere was $\geq 2$ and the absolute gene copy number was $\geq 10$. Low-level amplification was defined as a ratio of $\geq 2$ and an absolute gene copy number of $>4$ but $<10$. The remaining tumors were considered as not amplified.

\section{HER2 Immunohistochemistry}

For HER2 protein detection, the HercepTest kit (DAKO, Glostrup, DK) was used according to manufacturer's instructions. HER2 scoring was performed according to the 4 step-scale $(0,1+, 2+, 3+)$ outlined in the HercepTest manual for breast cancer.

\section{Large Section Validation/Heterogeneity Analysis}

To validate the results of the tissue microarray and to determine the heterogeneity of HER2 status, all available tumor tissue of individual high-level amplified cases was analyzed by ERBB2 FISH and HER2 immunohistochemistry. Between two and nine paraffin blocks containing tumor tissue were tested including up to three lymph-node metastasis per patient (Table 2). The complete tumor areas on large sections were screened for HER2 expression as well as presence or absence of ERBB2 amplification. The percentage of tumor areas with high-level and low-level amplification as well as of areas without amplification for each tumor was estimated 
Table 1 Relationship between ERBB2 amplification and HER2 expression and clinico-pathological parameters

\begin{tabular}{|c|c|c|c|c|c|c|c|c|c|c|c|}
\hline & & \multirow[b]{2}{*}{$\mathrm{n}$ on $T M A$} & \multicolumn{3}{|c|}{ ERBB2 FISH result } & \multirow[t]{2}{*}{$\mathrm{P}$-value } & \multicolumn{4}{|c|}{ HER2 immunohistochemistry result } & \multirow[t]{2}{*}{$\mathrm{P}$-value } \\
\hline & & & $\begin{array}{l}\mathrm{n} \\
\text { evaluable }\end{array}$ & $\begin{array}{l}\text { Low-level } \\
\text { amplification }\end{array}$ & $\begin{array}{l}\text { High-level } \\
\text { amplification }\end{array}$ & & $\underset{\text { evaluable }}{\mathrm{n}}$ & $1+$ & $2+$ & $3+$ & \\
\hline All tumors & & 590 & 526 & $7(1.3 \%)$ & $10(1.9 \%)$ & & 531 & $65(12.2 \%)$ & $40(7.5 \%)$ & $13(2.4 \%)$ & \\
\hline \multirow[t]{3}{*}{ Histology } & Adenocarcinoma & $219(37.1 \%)$ & 190 & $4(2.1 \%)$ & $8(4.2 \%)$ & $0.0008^{\mathrm{a}}$ & 193 & $35(18.1 \%)$ & $20(10.4 \%)$ & $7(3.6 \%)$ & $<0.0001^{\mathrm{a}}$ \\
\hline & $\begin{array}{l}\text { Squamous cell } \\
\text { carcinoma }\end{array}$ & $250(42.4 \%)$ & 227 & 0 & $1(0.4 \%)$ & & 227 & $18(7.9 \%)$ & $10(4.4 \%)$ & $1(0.4 \%)$ & \\
\hline & $\begin{array}{l}\text { Large cell } \\
\text { carcinoma }\end{array}$ & $121(20.5 \%)$ & 109 & $3(2.8 \%)$ & $1(0.9 \%)$ & & 111 & $12(10.8 \%)$ & $10(9.0 \%)$ & $5(4.5 \%)$ & \\
\hline \multirow[t]{3}{*}{ Grade $^{\mathrm{b}}$} & G1 & $14(2.4 \%)$ & 12 & 0 & 0 & 0.1036 & 13 & $1(7.7 \%)$ & $1(7.7 \%)$ & 0 & 0.03240 \\
\hline & G2 & $339(57.5 \%)$ & 302 & $3(1.0 \%)$ & $3(1.0 \%)$ & & 304 & $35(11.5 \%)$ & $19(6.3 \%)$ & $4(1.3 \%)$ & \\
\hline & G3 & $116(19.7 \%)$ & 103 & $1(1.0 \%)$ & $6(5.8 \%)$ & & 103 & $17(16.5 \%)$ & $10(9.7 \%)$ & $4(3.9 \%)$ & \\
\hline \multirow[t]{5}{*}{ Stage } & pT1 & $168(28.5 \%)$ & 145 & $1(0.7 \%)$ & $1(0.7 \%)$ & 0.4029 & 145 & $11(7.6 \%)$ & $11(7.6 \%)$ & 0 & 0.0180 \\
\hline & pT2 & $314(53.2 \%)$ & 287 & $4(1.4 \%)$ & $7(2.4 \%)$ & & 288 & $39(13.5 \%)$ & $25(8.7 \%)$ & $12(4.2 \%)$ & \\
\hline & pT3 & $50(8.5 \%)$ & 47 & 0 & $1(2.1 \%)$ & & 47 & $8(17.0 \%)$ & $2(4.3 \%)$ & $1(2.1 \%)$ & \\
\hline & pT4 & $50(8.5 \%)$ & 41 & $2(4.9 \%)$ & $1(2.4 \%)$ & & 45 & $6(13.3 \%)$ & $2(4.4 \%)$ & 0 & \\
\hline & pTX & $8(1.4 \%)$ & 6 & 0 & 0 & & 6 & $1(16.7 \%)$ & 0 & 0 & \\
\hline \multirow[t]{5}{*}{ Nodal stage } & pNo & $257(43.6 \%)$ & 223 & $2(0.9 \%)$ & $4(1.8 \%)$ & 0.0660 & 228 & $28(12.3 \%)$ & $18(7.9 \%)$ & $8(3.5 \%)$ & 0.0109 \\
\hline & $\mathrm{pN} 1$ & $159(26.9 \%)$ & 144 & $3(2.1 \%)$ & 0 & & 146 & $17(11.6 \%)$ & $6(4.1 \%)$ & 0 & \\
\hline & $\mathrm{pN} 2$ & $106(18.0 \%)$ & 100 & 0 & $4(4.0 \%)$ & & 99 & $16(16.2 \%)$ & $11(11.1 \%)$ & $4(4.0 \%)$ & \\
\hline & $\mathrm{pN} 3$ & $19(3.2 \%)$ & 17 & 0 & $1(5.9 \%)$ & & 18 & 0 & $1(5.6 \%)$ & $1(5.6 \%)$ & \\
\hline & pNX & $49(8.3 \%)$ & 42 & $2(4.8 \%)$ & $1(2.4 \%)$ & & 40 & $4(10.0 \%)$ & $4(10.0 \%)$ & 0 & \\
\hline Distant & pM1 & $33(5.6 \%)$ & 26 & 0 & 0 & 0.4163 & 27 & $3(11.1 \%)$ & 0 & 0 & 0.1119 \\
\hline metastases & pMX & $557(94.4 \%)$ & 500 & $7(1.4 \%)$ & $10(2.0 \%)$ & & 504 & $62(12.3 \%)$ & $40(7.9 \%)$ & $13(2.6 \%)$ & \\
\hline
\end{tabular}

${ }^{\mathrm{a}}$ Adenocarcinoma versus squamous cell carcinoma.

${ }^{b}$ All large cell carcinomas are exclusively considered as undifferentiated (G4).

independently by two experienced pathologists (TJG and AHM). For validation of the results obtained from tissue microarrays, 12 tumors with a positive HER2 immunohistochemistry result $(2+)$ but without $E R B B 2$ amplification were randomly selected. From these 12 patients, one large section of tumor tissue each was retested by FISH and immunohistochemistry.

\section{Statistical Analysis}

Contingency table analysis and $\chi^{2}$ (likelihood) test were used to study the relationship between ERBB2/ HER2 alterations and categorical parameters. The Kaplan-Meier method was used for survival analysis. For statistical analysis, the JMP 8.0 software (SAS Institute Inc, NC, USA) was used.

\section{Results}

\section{ERBB2 FISH}

ERBB2 gene copy numbers were interpretable in 526 arrayed tumor samples. Analysis failed in 64 tumors because hybridization quality was too low, not enough tumor cells were analyzable or the entire tissue spot was missing on the tissue microarray slide. ERBB2 amplification (defined as ratio ERBB2/ Cen $17 \geq 2$ and absolute ERBB2 signals >4) was found in $17(3 \%)$ cancers including $10(2 \%)$ tumors with a high-level amplification (ERBB2 gene copy number $\geq 10$ ). High-level ERBB2 amplification was significantly more frequent in adenocarcinomas (4\%) than in squamous cell carcinomas $(P=0.0051)$. Highlevel ERBB2 amplification was also significantly related to higher tumor grade (G3) in adenocarcinomas $(P=0.0238)$ resulting in a frequency of $11 \%$ of tumors with a high-level ERBB2 amplification within the subgroup of 57 high-grade (G3) adenocarcinomas. Twelve percent of high-grade adenocarcinomas showed any ERBB2 amplification (high- and lowlevel amplification). ERBB2 amplification was unrelated to primary tumor stage, nodal stage or presence of distant metastasis. All results are summarized in Table 1. Patients with ERBB2 amplified tumors had a significantly shortened survival as compared with patients with normal ERBB2 status in univariate analysis (Figure $1, P=0.03$ ) but not in multivariate analysis against the prognostic factors stage, nodal status and grade (Supplementary Table 1).

\section{HER2 Immunohistochemistry}

Immunohistochemical analysis of HER2 protein was successful in 531 tumors. A total of 59 samples were excluded from analysis because the tissue spots were missing on the tissue microarray slide or did not contain enough unequivocal tumor cells for evaluation. Positive immunostaining (including $2+$ and $3+$ ) was seen in $10 \%$ of tumors and was significantly more frequent in adenocarcinomas than in squamous cell carcinomas $(P=0.0016)$. Positivity was more frequent in high-grade than in low-grade tumors (Table 1). 
Table 2 Heterogeneity analysis of ERBB2 amplification

\begin{tabular}{|c|c|c|c|c|c|c|c|}
\hline \multirow[t]{2}{*}{ Case no. } & \multirow[t]{2}{*}{ Histology } & \multirow[t]{2}{*}{$T(\mathrm{n})^{a}$} & \multirow[t]{2}{*}{$L N(\mathrm{n})^{a}$} & \multicolumn{4}{|c|}{ ERBB2 FISH } \\
\hline & & & & $\begin{array}{c}\text { High-level } \\
\text { amplification (\%) }\end{array}$ & $\begin{array}{l}\text { Low-level } \\
\text { amplification }\end{array}$ & Negative & $\begin{array}{l}\text { ERBB2 signals } \\
\text { in high-level } \\
\text { amplified areas }\end{array}$ \\
\hline 1 & Adenocarcinoma & 3 & 2 & 100 & & & 40-50 (cluster) \\
\hline 2 & Adenocarcinoma & 4 & 0 & 85 & $15 \%$ (ratio 2.8 ) & & 10-25 (cluster) \\
\hline 3 & Adenocarcinoma & 4 & 0 & 100 & & & 15-30 (cluster) \\
\hline 4 & Large cell carcinoma & 3 & 0 & 10 & & $90 \%$ & 10-20 (cluster) \\
\hline 5 & Adenocarcinoma & 3 & 2 & 100 & & & 10-40 (cluster) \\
\hline 6 & Adenocarcinoma & 3 & 0 & 100 & & & 20-40 (cluster) \\
\hline 7 & Squamous cell carcinoma & 2 & 0 & 100 & & & 40-50 (cluster) \\
\hline 8 & Adenocarcinoma & 3 & 0 & 15 & & $85 \%$ & 30-50 (cluster) \\
\hline 9 & Adenocarcinoma & 6 & 3 & 10 & $90 \%$ (ratio 2.2 ) & & 10-20 (cluster) \\
\hline 10 & Adenocarcinoma & 3 & 0 & 100 & & & 10-20 (cluster) \\
\hline
\end{tabular}

${ }^{a}$ Analyzed tumor containing paraffin blocks of primary tumor (T) and lymph-node metastasis (LN).

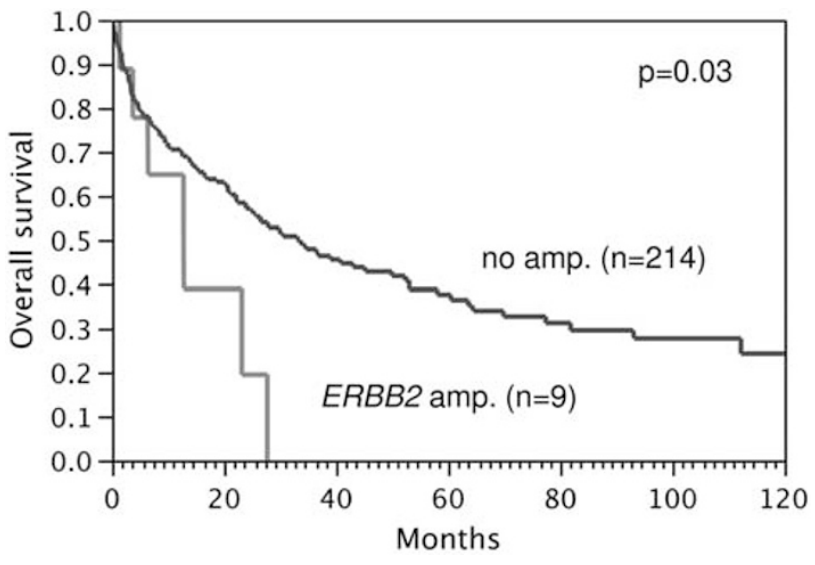

Figure 1 Association between ERBB2 amplification and patient survival (Kaplan-Meier survival analysis). The significance of amplification status on overall survival holds true only in univariate but not in multivariate analysis.

\section{ERBB2 Amplification versus HER2 Expression}

Both FISH and immunohistochemistry were interpretable on the same tissue spot in 516 lung cancers and could be matched. Expression and gene amplification were significantly associated $(P<0.0001$, Figure 2). The association was pronounced for highlevel amplified cases showing strong HER2 expression $(3+)$ in $70 \%$ of cases. Amplified tumors showed significant HER2 protein expression $(2+$ or $3+$ ) with the exception of four cases (two lowlevel and two high-level amplified cases). Only 4 of 39 tumors with a $2+$ HER2 immunostaining had gene amplification. For validation of these results, 12 cases with an HER2 $2+$ immunostaining but no gene amplification were retested by immunohistochemistry and FISH on one large section of tumor tissue each. In all cases at least focal-positive expression of the protein was observed in vicinity of the removed tissue core used for tissue microarray

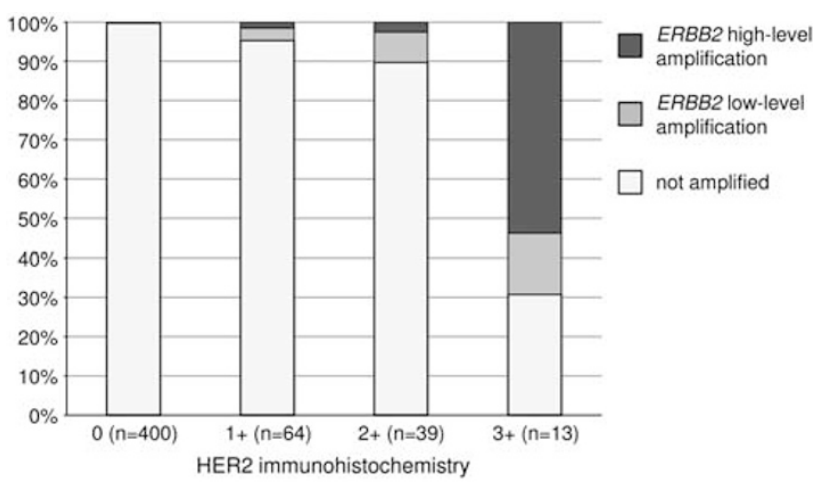

Figure 2 Association of HER2 expression and ERBB2 amplification.

construction. ERBB2 gene amplification was not found in any of these 12 cases.

\section{Heterogeneity of ERBB2 Amplification and HER2 Expression}

Ten tumors with high-level amplification recognized on the tissue microarray were selected for ERBB2 FISH analysis and HER2 immunohistochemistry of all available tumor tissue on large sections. $E R B B 2$ amplification was verified in all cases in the tumor area surrounding the tissue core used for tissue microarray construction. In six cases, a homogeneous high-level ERBB2 amplification could be demonstrated in all analyzed tumor cells. Two cases showed a heterogeneous pattern with highlevel and low-level amplified tumor areas and two cases showed non-amplified tumor next to highlevel amplified tumor areas. For one of these heterogeneous cases, three additional lymph node metastasis were available for ERBB2 analysis. This case showed high-level next to low-level amplification in the primary tumor while the metastasis showed homogeneous low-level amplification 

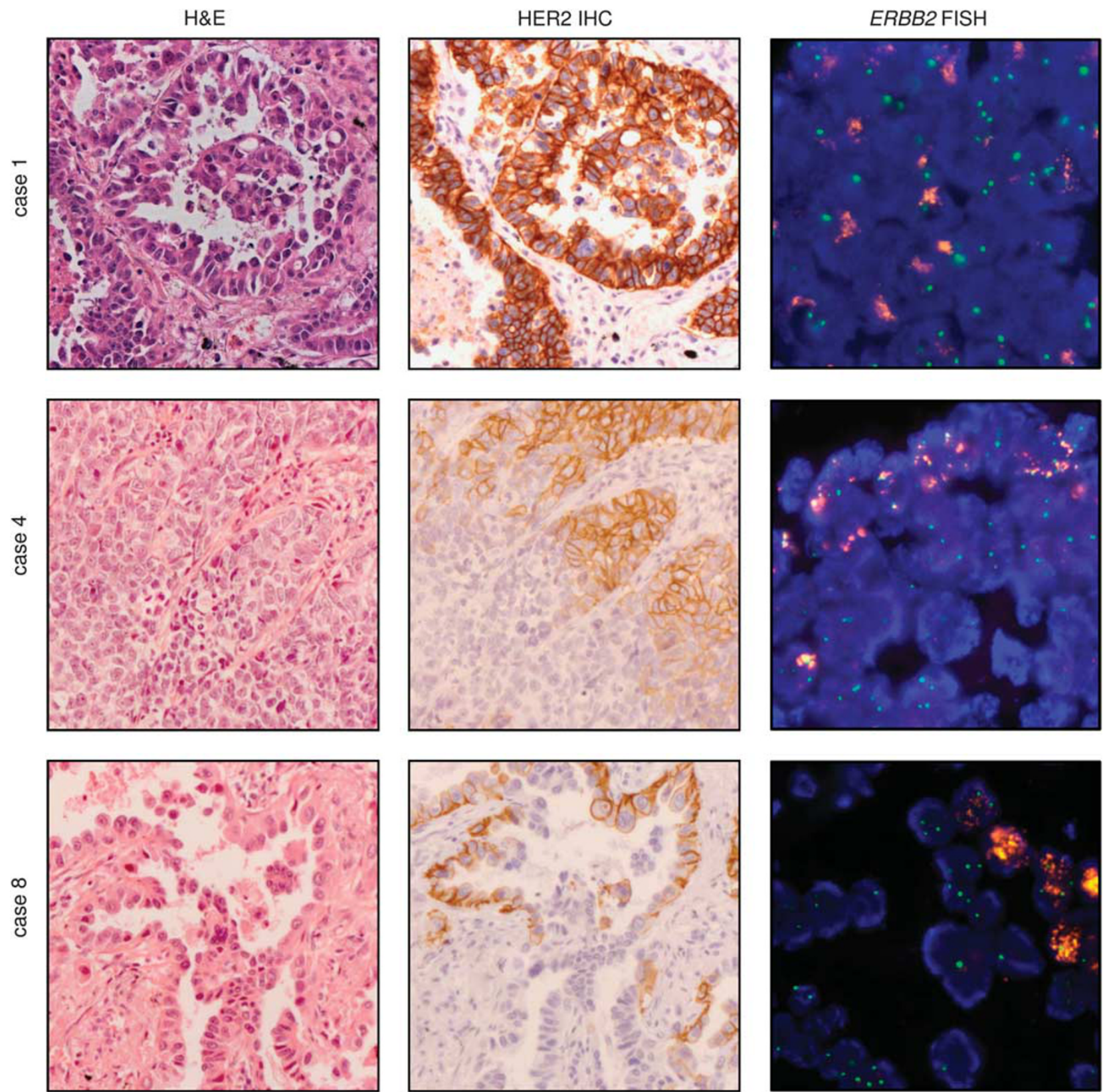

Figure 3 H\&E staining, HER2 immunohistochemistry and ERBB2 FISH analysis of tumors with ERBB2 amplification. Case 1 shows homogeneous strong membranous staining by immunohistochemistry. Corresponding FISH demonstrates ERBB2 amplification. The tumor cells exhibit clusters of orange signals (up to 50 per blue stained nucleus) indicating the ERBB2 gene. The green signals (1-2 per nucleus) represent the centromeric region of chromosome 17. Cases 4 and 8 show strong staining by immunohistochemistry only in a fraction of tumor cells. Corresponding FISH analyses display clusters of orange signals (ERBB2 gene) only in some tumor cells, while other tumor cells exhibit 1-3 orange signals, demonstrating heterogeneous gene amplification. The green signals (1-3 per nucleus) again represent the centromeric region of chromosome 17 .

(Table 2). Expression analyses were congruent with the FISH results in six cases (four homogeneously amplified and two amplified/non amplified cases). A strong membraneous staining $(3+)$ was observed in amplified tumor areas while no or weak staining $(0$ or $1+$ ) was found in non-amplified areas. Two homogenously amplified cases (cases 5 and 10 in Table 2) showed heterogeneous staining with only focal positivity. The two cases with high-level and low-level amplified tumor areas (cases 2 and 9) showed heterogeneous staining with only focal positivity not congruent with the FISH result. Histologic review of the heterogeneous tumors revealed no morphologic differences between tumor areas with different HER2 status (Figure 3).

\section{Discussion}

ERBB2 amplification was found in 17 (3\%) of 526 examined lung cancers including 10 cases $(2 \%)$ with 
high-level amplification. Amplification was particularly frequent in adenocarcinomas $(6 \%)$ and large cell carcinomas $(4 \%)$. The frequency observed in our study is in the range $(1-5 \%)$ of other studies performing ERBB2 FISH in lung cancer. ${ }^{11,13,15,16}$ The predilection of adenocarcinomas for ERBB2 amplification is in line with the results from earlier studies showing a correlation of HER2 expression with this histologic subtype. ${ }^{11}$ We did not observe significant associations with tumor stage or presence of metastases but found a significant link between ERBB2 amplification and poor prognosis although in univariate analysis only. A similar observation has been reported before. ${ }^{16}$ Within adenocarcinomas, ERBB2 amplification was particularly frequent in high-grade cancers. As a result, 7 of 57 grade 3 adenocarcinomas $(12 \%)$ were amplified, including 6 cases with high-level amplification. This subgroup obviously has the highest potential for anti-HER2 therapy.

HER2 positivity by immunohistochemistry was more frequently seen than gene amplification. In particular, a relatively high number of tumors with moderate HER2 expression $(2+)$ but without gene amplification were observed. Only $10 \%$ of tumors with a $2+$ immunohistochemistry result showed $E R B B 2$ gene amplification. Based on these data, one could conclude, that-in contrast to breast cancersome low-level HER2 overexpression may occur in lung cancer in the absence of gene amplification. It is also possible, however, that some of the positive immunohistochemistry staining represent artifacts. It is well known, that insufficient formalin fixation with consecutive exposure of the tissue to ethanol during technical processing can lead to false positive HER2 immunohistochemistry. ${ }^{28}$ On the other hand, false negative results of HER2 immunohistochemistry cannot always be excluded. Storage of paraffin material can result in reduced HER2 immunoreactivity. ${ }^{28}$ This might be the reason for focal HER2 negativity by immunohistochemistry in some ERBB2 amplified cases in our study as the paraffin material of these cases was stored between 7 and 18 years.

Presence of ERBB2 amplification is important for response to trastuzumab in breast and stomach cancer. However, not all tumors expressing a molecular drug target respond favorable to the corresponding medication. For example, only about $50 \%$ of HER2-positive breast cancers respond to trastuzumab. ${ }^{29,30}$ Heterogeneity of ERBB2 amplification and overexpression within a tumor and between primary tumors and their metastases is one potential cause for treatment failure as the primary tumor is typically removed from the patient and molecularly analyzed while the metastases remaining in the patient are subsequently being treated with the drug. Our data suggest that molecular heterogeneity may be more frequent in lung cancer than in breast cancer, at least for ERBB2. Our analysis of 41 different tumor blocks from 10 patients with high-level ERBB2 amplification in their lung cancer revealed heterogeneity of amplification within the primary tumor in 4 of 10 patients (Table 2). Although the number of cases analyzed is not large, these data suggest that ERBB2 amplification cannot be automatically considered as homogeneous if a test is positive in lung cancer. In breast cancer, heterogeneity within primary tumors and between primary tumors and their metastases is hardly seen. ${ }^{31}$ Presence of amplification restricted to subsets of cells in lung cancers argues for a role of ERBB2 amplification in lung cancer progression. In this context, it is interesting to note that 2 of 10 cancers had sub-populations with low-level amplification next to areas with high-level amplification.

Although HER2 overexpression and ERBB2 amplification clearly exist in lung cancer, clinical trials with trastuzumab were not successful. Two phase II studies did not find any convincing effect of trastuzumab treatment. ${ }^{18,19}$ The first study by Gatzemeier et al enrolled 103 patients with HER2positive lung cancers of different histologic subtypes (non-small cell lung cancer). In all, 17\% of 617 screened patients were found to be HER2 positive by immunohistochemistry $(2+, 3+)$ and were subsequently randomized. The second study by Langer et al included adenocarcinomas, squamous cell carcinomas and large cell undifferentiated carcinomas of the lung with detectable HER2 expression by immunohistochemistry $(1+, 2+$ or $3+$ ) which resulted in 59\% HER2-positive cases. Fifty-three patients were enrolled in this study. Although the authors of both studies could not show clinical benefit of trastuzumab treatment, both note that a potential benefit was seen in the subgroup of HER2 $3+$ tumors but that this group was too small in both studies to provide definitive information. Two smaller studies with similar inclusion criteria enrolling 21 and 24 patients could not see any relationship between HER2 expression and response. ${ }^{32,33}$ The small molecule lapatinib was recently also tested as monotherapy in adenocarcinoma and squamous cell carcinoma of the lung. ${ }^{34}$ Although the results did not show any significant number of tumor regressions, one of two patients with a retrospective tested ERBB2 amplification showed partial response to therapy.

The number of analyzed cases even within the present study is too low to provide a clear number of how many lung cancers have homogeneous highlevel ERBB2 gene amplification. From our data, this number may be in the range of $1 \%$. Even though a fraction of $1 \%$ positive cancers does not sound very high, it must be considered, that $>500000$ lung cancers are annually diagnosed alone in the United States and in Europe. ${ }^{35}$ Moreover, the relatively high prevalence of ERBB2 amplification in high-grade adenocarcinomas might justify further clinical evaluation of HER2 targeted therapy at least in this subgroup. 
In conclusion, a thorough analysis of 590 adenocarcinomas, squamous cell carcinomas and large cell undifferentiated carcinomas of the lung using FDAapproved reagents showed high-level ERBB2 gene amplification in $2 \%$ of these tumors with a strong preference for high-grade adenocarcinomas. Considering experiences with breast and gastric cancer, our data suggest that these lung cancer patients might be good candidates for a trastuzumab therapy. Clinical trials, which have been unsuccessful so far, have included between 17 and 59\% of screened patients based on immunohistochemical analyses. We therefore assume that a benefit of anti-HER2 medication on properly selected lung cancer patients is possible, based on the existing literature. From our data, it appears that $1 \%$ of lung cancer patients have homogeneous high-level ERBB2 amplification. Attempts to assess the utility of anti-HER2 therapy for these patients appear to be justified.

\section{Acknowledgement}

We appreciate the excellent technical support of Silvia Schnöger, Sascha Eghtessadi and Christina Koop.

\section{Disclosure/conflict of interest}

The authors declare no conflict of interest.

\section{References}

1 Hynes NE, Lane HA. ERBB receptors and cancer: the complexity of targeted inhibitors. Nat Rev Cancer 2005; 5:341-354.

2 Normanno N, Bianco C, Strizzi L, et al. The ErbB receptors and their ligands in cancer: an overview. Curr Drug Targets 2005;6:243-257.

3 Rabindran SK. Antitumor activity of HER-2 inhibitors. Cancer Lett 2005;227:9-23.

4 Cobleigh MA, Vogel CL, Tripathy D, et al. Multinational study of the efficacy and safety of humanized anti-HER2 monoclonal antibody in women who have HER2-overexpressing metastatic breast cancer that has progressed after chemotherapy for metastatic disease J Clin Oncol 1999;17:2639-2648.

5 Slamon DJ, Leyland-Jones B, Shak S, et al. Use of chemotherapy plus a monoclonal antibody against HER2 for metastatic breast cancer that overexpresses HER2. N Engl J Med 2001;344:783-792.

6 Vogel CL, Cobleigh MA, Tripathy D, et al. Efficacy and safety of trastuzumab as a single agent in first-line treatment of HER2-overexpressing metastatic breast cancer. J Clin Oncol 2002;20:719-726.

7 Bang YJ, Van Cutsem E, Feyereislova A, et al. Trastuzumab in combination with chemotherapy versus chemotherapy alone for treatment of HER2-positive advanced gastric or gastro-oesophageal junction cancer (ToGA): a phase 3, open-label, randomised controlled trial. Lancet 2010;376:687-697.
8 Geyer CE, Forster J, Lindquist D, et al. Lapatinib plus capecitabine for HER2-positive advanced breast cancer. N Engl J Med 2006;355:2733-2743.

9 Bunn Jr PA, Helfrich B, Soriano AF, et al. Expression of Her-2/neu in human lung cancer cell lines by immunohistochemistry and fluorescence in situ hybridization and its relationship to in vitro cytotoxicity by trastuzumab and chemotherapeutic agents Clin Cancer Res 2001;7:3239-3250.

$10 \mathrm{Au}$ NH, Cheang M, Huntsman DG, et al. Evaluation of immunohistochemical markers in non-small cell lung cancer by unsupervised hierarchical clustering analysis: a tissue microarray study of 284 cases and 18 markers. J Pathol 2004;204:101-109.

11 Hirsch FR, Varella-Garcia M, Franklin WA, et al. Evaluation of HER-2/neu gene amplification and protein expression in non-small cell lung carcinomas. Br J Cancer 2002;86:1449-1456.

12 Kwiatkowski DJ, Harpole Jr DH, Godleski J, et al. Molecular pathologic substaging in 244 stage I nonsmall-cell lung cancer patients: clinical implications J Clin Oncol 1998;16:2468-2477.

13 Nakamura H, Saji H, Ogata A, et al. Correlation between encoded protein overexpression and copy number of the HER2 gene with survival in non-small cell lung cancer. Int J Cancer 2003;103:61-66.

14 Cappuzzo F, Varella-Garcia M, Shigematsu H, et al. Increased HER2 gene copy number is associated with response to gefitinib therapy in epidermal growth factor receptor-positive non-small-cell lung cancer patients. J Clin Oncol 2005;23:5007-5018.

15 Pelosi G, Del Curto B, Dell'Orto P, et al. Lack of prognostic implications of HER-2/neu abnormalities in 345 stage I non-small cell carcinomas (NSCLC) and 207 stage I-III neuroendocrine tumours (NET) of the lung. Int J Cancer 2005;113:101-108.

16 Tan D, Deeb G, Wang J, et al. HER-2/neu protein expression and gene alteration in stage I-IIIA nonsmall-cell lung cancer: a study of 140 cases using a combination of high throughput tissue microarray, immunohistochemistry, and fluorescent in situ hybridization. Diagn Mol Pathol 2003;12:201-211.

17 Pellegrini C, Falleni M, Marchetti A, et al. HER-2/Neu alterations in non-small cell lung cancer: a comprehensive evaluation by real time reverse transcriptionPCR, fluorescence in situ hybridization, and immunohistochemistry. Clin Cancer Res 2003;9:3645-3652.

18 Gatzemeier U, Groth G, Butts C, et al. Randomized phase II trial of gemcitabine-cisplatin with or without trastuzumab in HER2-positive non-small-cell lung cancer. Ann Oncol 2004;15:19-27.

19 Langer CJ, Stephenson P, Thor A, et al. Trastuzumab in the treatment of advanced non-small-cell lung cancer: is there a role? Focus on Eastern Cooperative Oncology Group study 2598. J Clin Oncol 2004;22: 1180-1187.

20 Andersson J, Linderholm B, Bergh J, et al. HER-2/neu (c-erbB-2) evaluation in primary breast carcinoma by fluorescent in situ hybridization and immunohistochemistry with special focus on intratumor heterogeneity and comparison of invasive and in situ components. Appl Immunohistochem Mol Morphol 2004;12:14-20.

21 Marx AH, Burandt EC, Choschzick M, et al. Heterogenous high-level HER-2 amplification in a small subset of colorectal cancers. Hum Pathol 2010;41: 1577-1585. 
22 Sauter G, Moch H, Moore D, et al. Heterogeneity of erbB-2 gene amplification in bladder cancer. Cancer Res 1993;53:2199-2203.

23 Bilous M, Osamura RY, Ruschoff J, et al. HER-2 amplification is highly homogenous in gastric cancer. Hum Pathol 2010;41:304-305; author reply 4-5.

24 Hofmann M, Stoss O, Shi D, et al. Assessment of a HER2 scoring system for gastric cancer: results from a validation study. Histopathology 2008;52: 797-805.

25 Marx AH, Tharun L, Muth J, et al. HER-2 amplification is highly homogenous in gastric cancer. Hum Pathol 2009;40:769-777.

26 Kononen J, Bubendorf L, Kallioniemi A, et al. Tissue microarrays for high-throughput molecular profiling of tumor specimens. Nat Med 1998;4:844-847.

27 World Health Organization Classification of Tumors. In: Travis WD, Brambilla E, Müller-Hermelink HK, Harris CC(eds) Tumours of the Lung, Pleura, Thymus and Heart. IARC Press: Lyon; 2004, pp 9-124.

28 Sauter G, Lee J, Bartlett JM, et al. Guidelines for human epidermal growth factor receptor 2 testing: biologic and methodologic considerations. J Clin Oncol 2009; 27:1323-1333.

29 Adamo V, Franchina T, Adamo B, et al. Safety and activity of trastuzumab-containing therapies for the treatment of metastatic breast cancer: our long-term clinical experience (GOIM study). Ann Oncol 2007;18 (Suppl 6) vi11-vi15.
30 Dawood S, Gonzalez-Angulo AM, Peintinger F, et al. Efficacy and safety of neoadjuvant trastuzumab combined with paclitaxel and epirubicin: a retrospective review of the M. D. Anderson experience. Cancer 2007;110:1195-1200.

31 Simon R, Nocito A, Hubscher T, et al. Patterns of her-2/ neu amplification and overexpression in primary and metastatic breast cancer. J Natl Cancer Inst 2001;93: 1141-1146.

32 Clamon G, Herndon J, Kern J, et al. Lack of trastuzumab activity in nonsmall cell lung carcinoma with overexpression of erb-B2: 39810: a phase II trial of Cancer and Leukemia Group B. Cancer 2005;103:1670-1675.

33 Zinner RG, Glisson BS, Fossella FV, et al. Trastuzumab in combination with cisplatin and gemcitabine in patients with Her2-overexpressing, untreated, advanced non-small cell lung cancer: report of a phase II trial and findings regarding optimal identification of patients with Her2-overexpressing disease. Lung Cancer 2004;44:99-110.

34 Ross HJ, Blumenschein Jr GR, Aisner J, et al. Randomized phase II multicenter trial of two schedules of lapatinib as first- or second-line monotherapy in patients with advanced or metastatic non-small cell lung cancer. Clin Cancer Res 2010;16:1938-1949.

35 Ferlay J, Shin H, Bray F, et al. GLOBOCAN 2008 v1.2, Cancer Incidence and Mortality Worldwide: IARC CancerBase No 10 [Internet]; Lyon, France: International Agency for Research on Cancer 2010.

Supplementary Information accompanies the paper on Modern Pathology website (http://www.nature.com/ modpathol) 